Quim. Nova, Vol. 35, No. 9, 1854-1857, 2012

\title{
ISOLAMENTO DO TUNGSTÊNIO DA VOLFRAMITA DA MINA DE IGARAPÉ MANTEIGA (RONDÔNIA - BRASIL) POR LIXIVIAÇÃO ÁCIDA
}

\author{
Jéssica Frontino Paulino e Julio Carlos Afonso* \\ Departamento de Química Analítica, Instituto de Química, Universidade Federal do Rio de Janeiro, Av. Athos da Silveira Ramos, \\ 149, B1. A, 21941-909 Rio de Janeiro - RJ, Brasil \\ José Luiz Mantovano, Cláudio Augusto Vianna e José Waldemar Silva Dias da Cunha \\ Instituto de Engenharia Nuclear, Rua Hélio de Almeida, 75, 21941-906 Rio de Janeiro - RJ, Brasil
}

Recebido em 3/1/12; aceito em 15/6/12; publicado na web em 24/8/12

\begin{abstract}
RECOVERY OF TUNGSTEN FROM WOLFRAMITE FROM THE IGARAPÉ MANTEIGA MINE (RONDÔNIA - BRAZIL) VIA ACIDIC LEACHING. We report results of the efficiency of tungsten extraction from wolframite concentrate (containing $61.5 \mathrm{wt} \%$ $\mathrm{WO}_{3}$ ) from the Igarapé Manteiga mine (state of Rondônia, Brazil) through acid leaching with strong mineral acids at $100{ }^{\circ} \mathrm{C}$ and 400 $\mathrm{rpm}$ for $2-4 \mathrm{~h}$. $\mathrm{HCl}$ yielded insoluble matter containing the highest $\mathrm{WO}_{3}$ content $(90 \mathrm{wt} \%)$. This solid was dissolved in concentrated $\mathrm{NH}_{3(\text { aq) }}$ at $25^{\circ} \mathrm{C}$ and the insoluble matter filtrated. The filtrate was slowly evaporated. $70 \mathrm{wt} \%$ of the tungsten present in the starting concentrate material was recovered as ammonium paratungstate (APT).
\end{abstract}

Keywords: tungsten; wolframite; acid leaching.

\section{INTRODUÇÃO}

O tungstênio é um metal amplamente utilizado nas indústrias automobilística, mecânica, química e aeroespacial, entre outras. Tem o ponto de fusão mais alto dentre todos os metais $\left(3419^{\circ} \mathrm{C}\right)$; é menor apenas do que o do carbono. A densidade é elevada $\left(19,3 \mathrm{~g} / \mathrm{cm}^{3}\right)$ e tem alta resistência mecânica em altas temperaturas. ${ }^{1}$ Dentre os mais de vinte minerais de tungstênio, dois deles - scheelita (tungstato de cálcio, $\mathrm{CaWO}_{4}$ ) e volframita (tungstatos de ferro e manganês, [Fe,Mn] $\mathrm{WO}_{4}$ ) - têm importância comercial. Na volframita, o teor de Fe pode variar de 20 a $80 \%$ em massa. $^{2}$

Até 2005, a participação das reservas brasileiras de minério de tungstênio representava em média somente $0,39 \%$ do total mundial. No final de 2004, a retomada da mineração desse elemento estimulou pesquisas que resultaram na descoberta da mina mais importante do Brasil em operação atualmente, a mina de Igarapé Manteiga, em Ariquemes, Rondônia, de onde é extraído o minério para produção de concentrado de volframita. Trata-se da maior reserva nacional de minério de tungstênio, com 20.321 t de $\mathrm{WO}_{3}$ contido. A partir de 2006, as reservas brasileiras de minério de tungstênio contido passaram a representar cerca $1 \%$ do total mundial. ${ }^{3,4}$

Os minérios de tungstênio são britados, moídos e submetidos a processos de separação por flotação e por densidade, magnética e eletrostática. ${ }^{2,45}$ Obtém-se o chamado concentrado, que contém $60-70 \%$ em massa de $\mathrm{WO}_{3} \cdot{ }^{1,2} \mathrm{O}$ Brasil ainda não produz tungstênio metálico.

\section{Processamento químico do concentrado de tungstênio}

A rota convencional consiste na digestão do concentrado com soluções alcalinas concentradas sob temperatura e pressão elevadas. ${ }^{5-10}$ Esse processamento visa a remoção de impurezas insolúveis, como sílica, ferro e manganês do tungstênio (que se dissolve como $\left.\mathrm{WO}_{4}{ }^{2-}\right)$. $\mathrm{O}$ ácido túngstico $\left(\mathrm{H}_{2} \mathrm{WO}_{4}\right)$ é obtido pela acidificação da solução alcalina com $\mathrm{HCl}$. $\mathrm{O} \mathrm{H}_{2} \mathrm{WO}_{4(\mathrm{~s})}$ isolado é, então, dissolvido em $\mathrm{NH}_{3(\text { aq) }}$; a evaporação dessa solução em condições previamente otimizadas resulta nos cristais de paratungstato de amônio (APT),

\footnotetext{
*e-mail: julio@iq.ufrj.br
}

$\left(\mathrm{NH}_{4}\right)_{10} \mathrm{~W}_{12} \mathrm{O}_{41} \cdot 11 \mathrm{H}_{2} \mathrm{O}$, que é o composto mais utilizado como ponto de partida na manufatura dos produtos finais da cadeia produtiva do tungstênio. ${ }^{10} \mathrm{~A}$ cristalização do APT não tem como único objetivo a recuperação de tungstênio da solução, mas também é uma etapa de purificação, separando-o de impurezas mais solúveis. A fim de garantir a pureza do APT, somente $85-90 \% \mathrm{~m} / \mathrm{m}$ de tungstênio são normalmente recuperados durante o processo de cristalização desse sal. ${ }^{11} \mathrm{O}$ processamento do concentrado ainda pode ser feito através da fusão com $\mathrm{Na}_{2} \mathrm{CO}_{3}$ ou $\mathrm{NaOH}$, a elevadas temperaturas, $850-1000{ }^{\circ} \mathrm{C}$ e $500-600{ }^{\circ} \mathrm{C}$, respectivamente. ${ }^{12}$

As principais desvantagens das rotas alcalinas são o elevado consumo de reagentes, a elevada pressão durante a lixiviação, a necessidade do uso de materiais refratários e de um controle rigoroso da temperatura. ${ }^{13}$

Em outras rotas alternativas são usados ácidos minerais fortes $\left(\mathrm{HCl}, \mathrm{H}_{2} \mathrm{SO}_{4}\right.$ e $\mathrm{HNO}_{3}$ ), segundo as reações 1 a 3 :

$(\mathrm{Fe}, \mathrm{Mn}) \mathrm{WO}_{4}+2 \mathrm{HCl} \rightarrow \mathrm{H}_{2} \mathrm{WO}_{4}+(\mathrm{Fe}, \mathrm{Mn}) \mathrm{Cl}_{2}$

$(\mathrm{Fe}, \mathrm{Mn}) \mathrm{WO}_{4}+\mathrm{H}_{2} \mathrm{SO}_{4} \rightarrow \mathrm{H}_{2} \mathrm{WO}_{4}+(\mathrm{Fe}, \mathrm{Mn}) \mathrm{SO}_{4}$

$(\mathrm{Fe}, \mathrm{Mn}) \mathrm{WO}_{4}+2 \mathrm{HNO}_{3} \rightarrow \mathrm{H}_{2} \mathrm{WO}_{4}+(\mathrm{Fe}, \mathrm{Mn})\left(\mathrm{NO}_{3}\right)_{2}$

A forma monomérica do íon tungstato, $\mathrm{WO}_{4}{ }^{2-}$, só ocorre em $\mathrm{pH} \geq 6,2$, em soluções livres de agentes complexantes. ${ }^{14}$ Em soluções ácidas existem inúmeras espécies, com várias formas de polimerização. ${ }^{15}$ Essas espécies são pouco solúveis em acidez livre superior a $1 \mathrm{~mol} \mathrm{~L}{ }^{-1}$, ocorrendo a precipitação do $\mathrm{H}_{2} \mathrm{WO}_{4}$. É bem conhecida a insolubilidade do tungstênio(VI) em solução de $\mathrm{HCl}$ diluído (1-3 mol L $\left.{ }^{-1}\right)$, sendo classificado no grupo I de cátions. Por isso, o $\mathrm{HCl}$ é o ácido mais usado na abertura dos concentrados. ${ }^{8,9,11}$

A reação 1 pode ser conduzida em condições bem mais brandas do que as usadas nos procedimentos mais convencionais, em meio alcalino, visto que a temperatura (próxima a $\left.100{ }^{\circ} \mathrm{C}\right)$ e pressão $(1$ atm) empregadas são relativamente baixas, além de ser um processo economicamente viável quanto ao uso de reagentes. Produz-se uma mistura sólida que contém $\mathrm{H}_{2} \mathrm{WO}_{4}$ e resíduos também insolúveis. Dois parâmetros de operação são críticos: ${ }^{16}$ a granulometria do concentrado, que deve ser fina o bastante para assegurar que o ácido reaja completamente com a volframita, evitando emprego de excesso de 
ácido e a geração de grandes quantidades de resíduos finais de processo e, a agitação do meio reacional, que deve impedir que $\mathrm{o}_{2} \mathrm{WO}_{4}$ produzido não bloqueie a superfície da volframita que ainda não reagiu, reduzindo com isso o rendimento do processo. Atualmente, a rota ácida é empregada essencialmente quando o concentrado não contém quantidades apreciáveis de silicatos, arseniatos ou fosfatos, que dificultam a precipitação do tungstênio principalmente devido à formação de heteropoliânions $\left(\mathrm{SiW}_{12} \mathrm{O}_{40}{ }^{4-}, \mathrm{PW}_{12} \mathrm{O}_{40}{ }^{3-}, \mathrm{AsW}_{12} \mathrm{O}_{40}{ }^{3-}\right)$ solúveis em meio ácido. ${ }^{17}$

Após ser separada por filtração, a massa sólida é dissolvida em solução de $\mathrm{NaOH}$, para obtenção de $\mathrm{Na}_{2} \mathrm{WO}_{4}$ (solúvel), e os resíduos insolúveis são separados por filtração. $\mathrm{O} \mathrm{H}_{2} \mathrm{WO}_{4}$ pode ser obtido pela acidificação da solução com $\mathrm{HCl}$ ou, ainda, pela adição de $\mathrm{CaCl}_{2}$, para precipitação de $\mathrm{CaWO}_{4}$, sendo em seguida tratado com $\mathrm{HCl}$. O $\mathrm{H}_{2} \mathrm{WO}_{4(\mathrm{~s})}$ obtido pode ser calcinado para produção de $\mathrm{WO}_{3}$ ou, então, tratado com solução de $\mathrm{NH}_{3}$ para produção de APT. ${ }^{11,14,18-20} \mathrm{O}$ APT ainda pode ser obtido pela solubilização direta do resíduo da lixívia ácida em $\mathrm{NH}_{3 \text { (aq.) }}$. Frequentemente, molibdatos podem estar associados aos tungstatos e não podem ser removidos simplesmente por métodos físicos de separação. ${ }^{18}$ Isso significa que a metodologia de isolamento do tungstênio dos demais elementos presentes no lixiviado depende da composição química do concentrado processado.

Tendo em vista a grande aplicabilidade do tungstênio em diversas áreas da tecnologia industrial e o aumento considerável das reservas mapeadas do Brasil, sobretudo em razão do potencial mineral da volframita da mina de Igarapé Manteiga, é de extrema importância que sejam desenvolvidos tratamentos apropriados ao concentrado do minério dessa jazida. O metal é considerado estratégico no século $\mathrm{XXI} .{ }^{4} \mathrm{O}$ presente trabalho foi desenvolvido visando a proposição e o desenvolvimento de uma rota para o isolamento de tungstênio da volframita oriunda da mina de Igarapé Manteiga, a partir da digestão do concentrado em ácidos minerais, seguida do isolamento do tungstênio do resíduo insolúvel por dissolução deste em solução de amônia, como APT.

\section{PARTE EXPERIMENTAL}

\section{Concentrado de volframita}

Empregou-se um concentrado de volframita oriundo da mina de Igarapé Manteiga, Rondônia. O concentrado, com granulometria inferior a $0,106 \mathrm{~mm}$ foi submetido à análise quantitativa por fluorescência de raios X. Sua composição química $(\% \mathrm{~m} / \mathrm{m})$, expressa por meio da estequiometria do óxido correspondente para cada elemento, é apresentada na Tabela 1 .

\section{Lixiviação ácida}

$\mathrm{HCl}, \mathrm{H}_{2} \mathrm{SO}_{4}$ e $\mathrm{HNO}_{3}$ foram utilizados como agentes de lixiviação. Para a determinação da quantidade de ácido necessária para o processo levou-se em consideração a reação do ácido com os componentes em teor maior que $1 \% \mathrm{~m} / \mathrm{m}$ (de acordo com a análise química da Tabela 1). Foi utilizado um excesso de $10 \% \mathrm{~m} / \mathrm{m}$ do ácido em relação à quantidade estequiométrica. Todos os procedimentos foram feitos em triplicata. Esperava-se que o resíduo insolúvel resultante da digestão contivesse as mesmas quantidades iniciais de tungstênio $\left(\mathrm{WO}_{3}\right.$ ), precipitado como $\mathrm{H}_{2} \mathrm{WO}_{4}$ (cor amarela), além do nióbio $\left(\mathrm{Nb}_{2} \mathrm{O}_{5}\right)$, e parte do fósforo $\left(\mathrm{P}_{2} \mathrm{O}_{5}\right)$, que pode ser parcialmente solubilizado mediante tratamento com ácido a quente. A Tabela 2 mostra as relações ácido/concentrado para os três ácidos empregados. A concentração do ácido utilizada em cada experimento foi ajustada em 2,0 mol L-1. A massa de concentrado empregada nos experimentos foi $10 \mathrm{~g}$.
Tabela 1. Composição química $(\% \mathrm{~m} / \mathrm{m})^{*}$ do concentrado da mina de volframita da mina de Igarapé Manteiga

\begin{tabular}{cc}
\hline Elemento (como óxido) & $\% \mathrm{~m} / \mathrm{m}$ \\
\hline $\mathrm{WO}_{3}$ & $61,53 \pm 0,12$ \\
$\mathrm{FeO}$ & $19,17 \pm 0,06$ \\
$\mathrm{MnO}$ & $8,53 \pm 0,04$ \\
$\mathrm{Al}_{2} \mathrm{O}_{3}$ & $6,92 \pm 0,10$ \\
$\mathrm{~K}_{2} \mathrm{O}$ & $1,10 \pm 0,02$ \\
$\mathrm{P}_{2} \mathrm{O}_{5}$ & $1,05 \pm 0,04$ \\
$\mathrm{Nb}_{2} \mathrm{O}_{5}$ & $0,68 \pm 0,01$ \\
$\mathrm{CaO}$ & $0,47 \pm 0,01$ \\
$\mathrm{SO}_{3}$ & $0,28 \pm 0,02$ \\
$\mathrm{TiO}_{2}$ & $0,16 \pm 0,02$ \\
$\mathrm{Bi}_{2} \mathrm{O}_{3}$ & $0,12 \pm 0,02$ \\
$\mathrm{Total}$ & 100,01 \\
\hline
\end{tabular}

*Obtida a partir da análise de 3 amostras distintas do concentrado

Tabela 2. Relação ácido/concentrado para os três ácidos empregados

\begin{tabular}{ccc}
\hline $\begin{array}{c}\text { Ácido } \\
\left(2 \mathrm{~mol} \mathrm{~L}^{-1}\right)\end{array}$ & $\begin{array}{c}\text { Razão mássica ácido/ } \\
\text { concentrado }(\mathrm{m} / \mathrm{m})\end{array}$ & $\begin{array}{c}\text { Razão volume ácido/massa de } \\
\text { concentrado }\left(\mathrm{mL} \mathrm{g}^{-1}\right)\end{array}$ \\
\hline $\mathrm{HCl}$ & $0,8: 1$ & $0,7: 1$ \\
$\mathrm{H}_{2} \mathrm{SO}_{4}$ & $1,1: 1$ & $0,6: 1$ \\
$\mathrm{HNO}_{3}$ & $1,4: 1$ & $1: 1$ \\
\hline
\end{tabular}

Os experimentos foram conduzidos em um balão de fundo chato sobre placa agitadora-aquecedora em capela. A velocidade da agitação (magnética) foi fixada em $400 \mathrm{rpm}$, a temperatura em 100 ${ }^{\circ} \mathrm{C}$ e o tempo pré-estabelecido foi de $2 \mathrm{~h}$. Ao balão foi acoplado um condensador de refluxo, para que se mantivesse um volume total constante da mistura reacional. Uma vez finalizado o experimento, filtrou-se imediatamente a solução em cadinho de vidro sinterizado sob vácuo; a massa insolúvel em ácido, de tonalidade amarela, foi lavada com água ( $\left.4 \mathrm{~mL} \mathrm{~g}^{-1}\right)$, até que a água de lavagem apresentasse $\mathrm{pH} \sim 6$, seca em estufa a $150^{\circ} \mathrm{C}$ por $3 \mathrm{~h}$, sendo resfriada e conservada em dessecador. As águas de lavagem foram incorporadas à solução ácida original. A massa insolúvel foi pesada e analisada para determinação de sua composição química.

Uma nova série de ensaios (em triplicata) foi realizada utilizando-se $\mathrm{HCl}$ (o ácido mais citado na literatura) para esse fim. A temperatura foi mantida em $100^{\circ} \mathrm{C}$ e a velocidade da agitação permaneceu em 400 rpm. A concentração de $\mathrm{HCl}$ variou entre 2 e $6 \mathrm{~mol} \mathrm{~L}^{-1}$ e os tempos de reação variaram de 2 e 4 h para cada valor de concentração do ácido.

\section{Isolamento do tungstênio}

A massa insolúvel contendo $\mathrm{H}_{2} \mathrm{WO}_{4}$ foi tratada com $\mathrm{NH}_{3(\mathrm{aq})}$ concentrado. $\mathrm{O}$ experimento foi conduzido em um béquer sobre placa agitadora na capela. A agitação (magnética) foi estabelecida em 200 rpm, à temperatura ambiente $\left(\sim 25^{\circ} \mathrm{C}\right)$. A solução amoniacal foi adicionada lentamente até total dissolução do sólido amarelo, restando ao final somente um resíduo de coloração acinzentada. A mistura foi deixada em repouso até decantação do resíduo, que foi filtrado em papel de filtro e lavado com solução de $\mathrm{NH}_{3} 6 \mathrm{~mol} \mathrm{~L}^{-1}$ (as águas de lavagem foram incorporadas à solução amoniacal). Após secagem em estufa a $150{ }^{\circ} \mathrm{C}$ por $3 \mathrm{~h}$ a massa foi resfriada, pesada e analisada quanto à composição química. A solução amoniacal foi mantida sob aquecimento (placa aquecedora em capela), com temperatura em 
torno de $40{ }^{\circ} \mathrm{C}$, para redução progressiva do volume da solução e cristalização de APT. A evaporação foi mantida até a secura e, então, o sólido foi pesado e analisado por FRX.

\section{Métodos analíticos}

O concentrado de volframita e todos os sólidos obtidos durante seu processamento foram analisados por fluorescência de raios $\mathrm{X}$ (FRX) em um espectrômetro de fluorescência de raios X com energia dispersiva, marca Shimadzu EDX 800HS. As amostras foram analisadas sobre filme de polipropileno. Curvas de calibração (0,1-1000 $\mathrm{mg} \mathrm{kg}^{-1}$ ) para W, Fe, Mn, Nb, Al, K, P, Ca, Ti e Bi foram empregadas para a dosagem quantitativa desses elementos.

\section{RESULTADOS E DISCUSSÃO}

\section{Eficiência dos ácidos na abertura do concentrado}

Os dados da Tabela 3 mostram que, dentre os três ácidos utilizados, o $\mathrm{HCl}$ foi o mais eficiente para o processamento do concentrado de volframita, cujo resíduo insolúvel é o mais rico em $\mathrm{WO}_{3}$. O resíduo final resultante do tratamento com $\mathrm{HCl}$ representou em média $83 \%$ da massa inicial, enquanto os resíduos do tratamento com $\mathrm{H}_{2} \mathrm{SO}_{4}$ e $\mathrm{HNO}_{3}$ representaram, respectivamente, 88 e $92 \% \mathrm{~m} / \mathrm{m}$. A massa insolúvel tem coloração diferente segundo o meio ácido empregado: amarelo limão $\left(\mathrm{HCl}\right.$, correspondendo ao $\mathrm{H}_{2} \mathrm{WO}_{4}$ ou $\left.\mathrm{WO}_{3} \cdot \mathrm{H}_{2} \mathrm{O}\right)$, pardo $\left(\mathrm{H}_{2} \mathrm{SO}_{4}\right)$ e cinza $\left(\mathrm{HNO}_{3}\right)$, que é a coloração do concentrado original. Isso sugere que os ácidos $\mathrm{H}_{2} \mathrm{SO}_{4}$ e $\mathrm{HNO}_{3}$ foram menos eficientes na decomposição da volframita. Os ácidos nítrico e sulfúrico são mais empregados quando o concentrado contém silicatos, fosfatos e arseniatos. ${ }^{11,18}$ Nos dados da literatura, o rendimento na recuperação de tungstênio através de lixiviação do concentrado em meio ácido é bastante variável. Em concentrados com teor médio de $60-70 \%$ $\mathrm{m} / \mathrm{m}$, o rendimento pode variar de 40 (usando $\left.\mathrm{H}_{2} \mathrm{SO}_{4}\right)^{20}$ a $99 \% \mathrm{~m} / \mathrm{m}$ (usando $\mathrm{HCl})^{14}$ de acordo com as condições experimentais empregadas, principalmente temperatura e pressão, e com a composição e granulometria do concentrado. De fato, a ausência ou a ocorrência de apenas uma pequena quantidade de elementos formadores de ânions, que formam sais insolúveis em meio ácido ( $\mathrm{Si}, \mathrm{As}, \mathrm{P}, \mathrm{Nb}$ ) com ferro e manganês, no concentrado de Igarapé Manteiga pode explicar ao menos em parte a diferença de comportamento do $\mathrm{HCl}$ frente aos os outros dois ácidos empregados neste estudo.

Estudos complementares com o $\mathrm{HCl}$ indicaram que um aumento da concentração do reagente e do tempo de reação não elevaram de

Tabela 3. Composição química $(\% \mathrm{~m} / \mathrm{m})$ das massas insolúveis resultantes da abertura ácida do concentrado de volframita

\begin{tabular}{cccc}
\hline \multirow{2}{*}{$\begin{array}{c}\text { Elemento } \\
\text { (como óxido) }\end{array}$} & \multicolumn{3}{c}{$\% \mathrm{~m} / \mathrm{m}$} \\
\cline { 2 - 4 } & $\mathrm{HCl}\left(2 \mathrm{~mol} \mathrm{~L}^{-1}\right)$ & $\mathrm{H}_{2} \mathrm{SO}_{4}\left(2 \mathrm{~mol} \mathrm{~L}^{-1}\right)$ & $\mathrm{HNO}_{3}\left(2 \mathrm{~mol} \mathrm{~L}^{-1}\right)$ \\
\hline $\mathrm{WO}_{3}$ & $90,30 \pm 0,15$ & $78,08 \pm 0,14$ & $70,43 \pm 0,13$ \\
$\mathrm{Fe}_{2} \mathrm{O}_{3}$ & $4,03 \pm 0,03$ & $13,10 \pm 0,05$ & $14,68 \pm 0,05$ \\
$\mathrm{MnO}$ & $1,72 \pm 0,02$ & $6,02 \pm 0,04$ & $6,73 \pm 0,04$ \\
$\mathrm{Al}_{2} \mathrm{O}_{3}$ & $\mathrm{ND}^{*}$ & $\mathrm{ND}$ & $4,39 \pm 0,09$ \\
$\mathrm{P}_{2} \mathrm{O}_{5}$ & $1,82 \pm 0,05$ & $1,21 \pm 0,05$ & $1,19 \pm 0,04$ \\
$\mathrm{Nb}_{2} \mathrm{O}_{5}$ & $1,06 \pm 0,01$ & $0,90 \pm 0,01$ & $0,79 \pm 0,01$ \\
$\mathrm{SO}_{3}$ & $0,60 \pm 0,02$ & $0,39 \pm 0,02$ & $0,37 \pm 0,02$ \\
$\mathrm{~K}_{2} \mathrm{O}$ & $0,44 \pm 0,02$ & $0,31 \pm 0,02$ & $0,35 \pm 0,02$ \\
\hline $\mathrm{Total}^{2}$ & 99,97 & 100,01 & 98,93 \\
\hline
\end{tabular}

\footnotetext{
* Não detectado
}

forma significativa o percentual mássico de $\mathrm{WO}_{3}$ na massa insolúvel resultante do processo de abertura, ou seja, essas variáveis não influenciaram significativamente no rendimento final desta etapa do processamento com base nas características do concentrado processado (composição química e granulometria).

\section{Isolamento e purificação do tungstênio}

O APT, sólido branco, obtido da purificação do $\mathrm{H}_{2} \mathrm{WO}_{4}$ pela solubilização da massa insolúvel em $\mathrm{NH}_{3(\text { aq.) }}$ concentrado, possuía em média $86,27 \pm 0,16 \% \mathrm{~m} / \mathrm{m}$ de $\mathrm{WO}_{3}$, contendo pequenas quantidades de enxofre, nióbio e ferro, de acordo com a Tabela 4. A Equação 4 representa o processo:

$12 \mathrm{H}_{2} \mathrm{WO}_{4}+10 \mathrm{NH}_{4} \mathrm{OH} \rightarrow\left(\mathrm{NH}_{4}\right)_{10} \mathrm{~W}_{12} \mathrm{O}_{41} \cdot 11 \mathrm{H}_{2} \mathrm{O}+6 \mathrm{H}_{2} \mathrm{O}$

Tabela 4. Composição média do APT obtido

\begin{tabular}{cc}
\hline Elemento (como óxido) & $\% \mathrm{~m} / \mathrm{m}$ \\
\hline $\mathrm{WO}_{3}{ }^{*}$ & $86,27 \pm 0,16$ \\
$\mathrm{SO}_{3}$ & $0,37 \pm 0,02$ \\
$\mathrm{Nb}_{2} \mathrm{O}_{5}$ & $0,21 \pm 0,01$ \\
$\mathrm{Fe}_{2} \mathrm{O}_{3}$ & $0,11 \pm 0,01$ \\
\hline
\end{tabular}

* valor teórico para o APT: $86,65 \% \mathrm{~m} / \mathrm{m}$

Com base no teor de $\mathrm{WO}_{3}$ no concentrado (Tabela 1) e na estequiometria $12 \mathrm{WO}_{3}=1\left(\mathrm{NH}_{4}\right)_{10} \mathrm{~W}_{12} \mathrm{O}_{41} \cdot 11 \mathrm{H}_{2} \mathrm{O}$, para um rendimento de $100 \%$, ter-se-ia uma massa de 7,16 g de APT para $10 \mathrm{~g}$ de concentrado. A massa obtida foi 5,01 $\pm 0,08 \mathrm{~g}$, indicando que $70 \% \mathrm{~m} / \mathrm{m}$ do tungstênio presente no concentrado foi recuperado neste produto. De fato, apesar da obtenção de um produto final com grau de pureza muito bom (Tabela 4), os resultados da análise química do resíduo insolúvel (acinzentado) em $\mathrm{NH}_{3(\mathrm{aq})}$ obtido a partir da dissolução da massa insolúvel em $\mathrm{HCl}$ (Tabela 5) confirmam que uma parcela considerável do $\mathrm{WO}_{3}$ inicial não foi recuperada. Cerca de $40 \%$ da massa insolúvel em $\mathrm{HCl}$ não foi solubilizada em $\mathrm{NH}_{3}$ e possui composição semelhante àquela da amostra original (Tabela 1). Isso indica que parte dos tungstatos de ferro e de manganês não reagiu. Se um tempo de reação mais longo e uma maior concentração de $\mathrm{HCl}$ não aumentaram o teor de tungstênio no resíduo insolúvel nesse ácido, a granulometria do concentrado deve ser reavaliada, pois a decomposição parcial da volframita pode ser atribuída ao tamanho da partícula que, neste caso, pode ser considerado relativamente grande.

Tabela 5. Composição química do resíduo insolúvel em $\mathrm{NH}_{3 \text { (aq) }}$

\begin{tabular}{cc}
\hline Elemento (como óxido) & $\% \mathrm{~m} / \mathrm{m}$ \\
\hline $\mathrm{WO}_{3}$ & $60,00 \pm 0,12$ \\
$\mathrm{Fe}_{2} \mathrm{O}_{3}$ & $16,81 \pm 0,05$ \\
$\mathrm{MnO}$ & $7,60 \pm 0,04$ \\
$\mathrm{Al}_{2} \mathrm{O}_{3}$ & $9,73 \pm 0,11$ \\
$\mathrm{Nb}_{2} \mathrm{O}_{5}$ & $2,59 \pm 0,01$ \\
$\mathrm{~K}_{2} \mathrm{O}$ & $1,80 \pm 0,03$ \\
$\mathrm{P}_{2} \mathrm{O}_{5}$ & $1,00 \pm 0,06$ \\
$\mathrm{TiO}_{2}$ & $0,34 \pm 0,02$ \\
$\mathrm{Bi}_{2} \mathrm{O}_{3}$ & $0,22 \pm 0,01$ \\
\hline $\mathrm{Total}$ & 100,09 \\
\hline
\end{tabular}

\section{CONCLUSÕES}

O processamento do concentrado de volframita da mina de 
Igarapé Manteiga por abertura com ácidos minerais fortes foi mais eficiente com ácido clorídrico, que promoveu a concentração do tungstênio $\left(\mathrm{WO}_{3}\right)$ em até $90,30 \% \mathrm{~m} / \mathrm{m}$, no resíduo insolúvel, antes da etapa de purificação. No entanto, apesar de proporcionar condições menos drásticas de operação, quando comparada aos processamentos pela via alcalina citados na literatura, a reação do tungstênio presente no resíduo insolúvel resultante da lixiviação ácida com amônia não foi completa, pois parte dele ainda permaneceu na forma de tungstato de ferro e manganês. Cerca de $70 \% \mathrm{~m} / \mathrm{m}$ do tungstênio inicial foram recuperados na forma de APT com elevada pureza.

\section{AGRADECIMENTOS}

J. F. Paulino agradece à CAPES pela bolsa de mestrado. Ao CNPq pelo auxílio financeiro.

\section{REFERÊNCIAS}

1. Barboza, F. L. M.; Perfil Analítico do Tungstênio, Boletim 24, DNPM: Rio de Janeiro, 1973.

2. Shedd, K. B.; Open-File Report 2005-2008, The U.S. Geological Survey: Washington, 2005.

3. Cano, T. M.; Costa, J. L.; Nesi, J. R.; Tungstênio. Economia Mineral do Brasil, DNPM: Rio de Janeiro, 2009.

4. Cano, T. M.; Sumário Mineral: Tungstênio, DNPM: Rio de Janeiro, 2010.
5. Zbranek, V.; Zbranek, Z.; Burnham, D. A.; US pat. 4,092,400 1978.

6. Queneau, P. B.; Huggins, D. K.; Beckstead, L. W.; US pat. 4,320,096 1982.

7. Quatrini, L. R.; Martin, B. E.; US pat. 4,353,879 1982.

8. Lassner, E.; Int. J. Refract. Met. Hard Mater. 1995, 13, 35.

9. Abdel-Rehim, A. M.; J. Therm. Anal. Calorim. 2001, 64, 1283.

10. Hairunnisha, S.; Sendil, G. K.; Rethinaraj, J. P.; Srinivasan, G. N.; Adaikkalam, P.; Kulandaisamy, S.; Hydrometallurgy 2007, 85, 67.

11. Luo, L.; Miyazaki, T.; Shibayama, A.; Yen, W.; Fujita, T.; Miner. Eng. 2003, 16, 665

12. Amer, A. M.; Hydrometallurgy 2000, 58, 251.

13. Menéndez, C. J.; Nolasco, E. J.; Tavani, E. L.; Pereira, E.; Can. Metall. Q. 1998, 37, 379 .

14. Koutsospyros, A.; Braida, W.; Christodoulatos, C.; Dermatas, D.; Strigul, N.; J. Hazard. Mater. 2006, 136, 1.

15. Smith, B. J.; Patrick, V. A.; Aust. J. Chem. 2000, 53, 965.

16. Zhao, Z.; Li, J.; Wang, S.; Li, H.; Liu, M.; Sun., P.; Li, Y.; Hydrometallurgy 2011, 108, 152 .

17. Goddard, J. B.; US pat. 4.346 .0611982$.

18. Kasey, J. B.; The Patent Office - London 860,890 1961.

19. General Eletric Company; The Patent Office - London 925,466 1963.

20. Sherrit Gordon Mines Limited; The Patent Office - London 987,340 1965. 